\title{
Diet, nutritional status and school performance among adolescents in Gaza Strip
}

A. Abudayya, ${ }^{1}$ Z. Shi, ${ }^{1}$ Y. Abed ${ }^{2}$ and G. Holmboe-Ottesen ${ }^{1}$

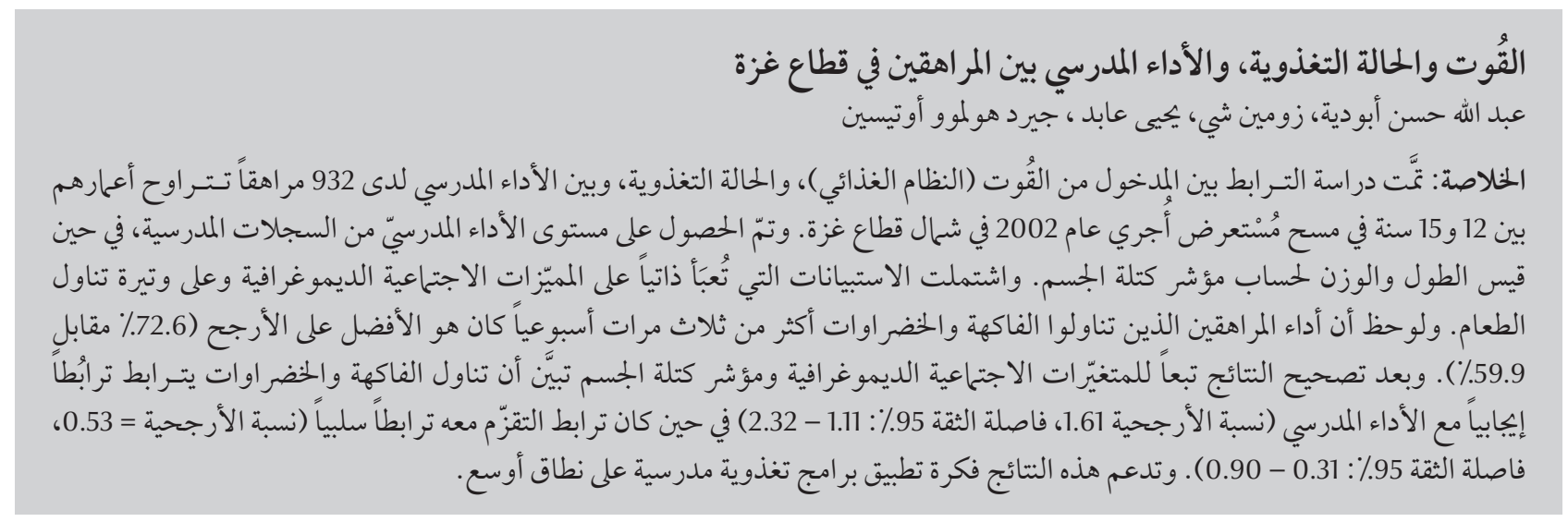

ABSTRACT The associations between dietary intake, nutritional status and school performance among 932 adolescents aged 12-15 years were examined in a cross-sectional survey in 2002 in north Gaza Strip. School performance was obtained from school records and height and weight were measured for body mass index (BMI). Self-administered questionnaires included sociodemographic characteristics and food frequency intakes. Adolescents consuming fruit and vegetables more than 3 times per week were more likely to have good school performance (72.6\% versus 59.9\%). When adjusting for sociodemographic variables and BMI, fruit and vegetables intake was positively associated with school performance $(\mathrm{OR}=1.61,95 \% \mathrm{Cl}$ : $1.11-2.32)$ and stunting was negatively associated $(\mathrm{OR}=0.53,95 \% \mathrm{Cl}$ : $0.31-0.90)$. The findings support a broader implementation of school nutrition programmes.

\begin{abstract}
Alimentation, état nutritionnel et résultats scolaires chez des adolescents de la Bande de Gaza
RÉSUMÉ L'association entre l'apport alimentaire, l'état nutritionnel et les résultats scolaires chez 932 adolescents âgés de 12 à 15 ans a été examinée dans une étude transversale conduite en 2002, dans le nord de la Bande de Gaza. Les résultats scolaires ont été obtenus à partir des dossiers scolaires. La taille et le poids ont été mesurés pour déterminer l'indice de masse corporelle. Les questionnaires auto-administrés portaient notamment sur les caractéristiques sociodémographiques et la fréquence des apports alimentaires. Les adolescents consommant des fruits et des légumes plus de trois fois par semaine étaient plus susceptibles d'avoir de bons résultats scolaires (72,6 \% contre 59,9\% pour ceux en consommant moins souvent). Après ajustement pour les variables sociodémographiques et l'indice de masse corporelle, I'apport en fruits et légumes était positivement associé aux résultats scolaires $(O R=1,61$; IC à $95 \%$ : $1,11-2,32)$ alors que le retard de croissance y était négativement associé $(O R=0,53$, IC à $95 \%: 0,31-0,90)$. Ces résultats justifient une mise en œuvre plus vaste des programmes de nutrition scolaire.
\end{abstract}

'Department of Community Medicine, Institute of Health and Society, University of Oslo, Oslo, Norway (Correspondence to A. Abudayya: abdallah.abudayya@medisin.uio.no; abudayya@gmail.com).

${ }^{2}$ School of Public Health, Al Quds University, Gaza City, Palestine.

Received: 12/07/09; accepted: 13/09/09 


\section{Introduction}

Performance in school is affected by a number of factors, including the quality of the school, the characteristics of the student's family such as socioeconomic status (SES) and parents' educational level, and the characteristics of the child [1].

The contribution of diet to students academic performance has rarely been reported [2,3], and the focus of much of the relevant research has been on the association between indicators of malnutrition and cognition [2]. Insufficient daily consumptions of foods has been found to affect health status, and poor health and nutritional status may hinder a child's ability to learn [4]. Moreover, nutritional deficiencies in childhood and adolescence can affect later behavioural development and the acquisition of social skills [5]. There is evidence that early childhood malnutrition, stunting, underweight, and anaemia are negatively associated with cognitive development, appropriate behaviour and academic attainment in later childhood [6]. Chronically undernourished children fare worse academically and fall behind in class; they are irritable and have difficulty concentrating, which can interfere with learning [7].In a study of Israeli girls, Kaufmann et al. observed that $3 \%$ of the girls aged 14 years and $11 \%$ of those aged 19 years had undesirable physical and mental side-effects from dieting, including impaired school performance [8].

One recent study from Canada found a positive association between intake of fruits and vegetables and school performance [2], but similar data are not available from other countries. The objective of our study was to describe the association between eating behaviour, especially intake of fruits and vegetables, and nutritional status and school performance among schoolchildren aged $12-15$ years in north Gaza Strip.

\section{Methods}

\section{Study design and setting}

This cross-sectional study was conducted in urban and suburban preparatory schools in north Gaza Strip during the fall of 2002. The design of this study has been described previously $[9,10]$. Briefly, the study was conducted in Gaza city, Jabalia village and the Jabalia refugee camp. In Gaza city, a high-income neighbourhood and a low-income neighbourhood were selected. The high-income area was characterized by a higher housing standard, better infrastructure and lower population density than the low-income neighbourhood. The selected communities covered both urban and semiurban areas, with Jabalia village at the semiurban end of the spectrum. The communities are in close proximity and can be considered as one continuous community due to the high population density.

\section{Sample}

A multistage cluster sampling technique was employed. In Gaza city a total of 6 schools were randomly selected: 4 were situated downtown in a high income area (Remal), of which 2 were government schools and 2 were run by the United Nations Reliefand Works Agency for Palestine Refugees (UNRWA); 2 other schools, both government, were selected from a low-income area of the city. In Jabalia village 2 government-run schools were randomly selected. Finally, in Jabalia refugee camp, 2 schools were selected, both UNRWA schools. Of the 2 schools in each area, 1 boys' school and 1 girls' school were selected. In each of the 10 schools, 3 classes were randomly selected to represent the 7 th, 8 th and 9th grades. Thus, students from 30 classes in 10 schools were invited to join the study. The age range of the study population was $12-15$ years.

\section{Data collection}

A self-administered, precoded questionnaire was filled in by the students in the classroom (time for completion was 45 minutes or less) in the presence of the researcher, who collected the questionnaires after they had been completed. Before the students began, the researcher explained all the questions. The students were instructed that answering the anonymous questionnaire was entirely voluntary and that the information would be treated confidentially. The questionnaire had been pilot-tested and adjusted prior to the actual survey. The students' dates of birth were obtained from the school records.

Demographic data were analysed in the following categories: age group [12, 13,14 and 15 years (i.e. $11.5-<12.5$ years, etc.)]; residential area based on the location of the school (high income; low income; village; refugee camp); father's current job status (unemployed; employed); father's educational level (low, $\leq 11$ years; medium, $12-14$ years; high, $15+$ years); and mother's educational level (low, $\leq 11$ years; medium/ high, $12+$ years). A household SES score based on the possession of household amenities was developed. A value of 1 was assigned to the ownership of 6 items (car, video/DVD player, television, satellite antenna, computer, refrigerator) and the sum was designated as the SES score (low, $\leq 2$; medium, 3-4; high, 5-6).

School performance during the previous year was obtained from school records. Good school performance was defined as an overall average grade of > $70 \%$ during the previous academic year.

Students were weighed without shoes and minimum clothes to the nearest $100 \mathrm{~g}$ using a weighing scale (Seca Selecta 791), and their height was measured barefoot to the nearest $0.1 \mathrm{~cm}$ using a stadiometer (Seca Bodymeter 208). Body mass index (BMI) categories (weight in $\mathrm{kg}$ divided by height in $\mathrm{m}^{2}$ ) were defined using National Center for Health Statistics and World Health Organization (NCHS/WHO) references for age and sex [11]: underweight 
(BMI < 5th percentile); normal weight (BMI 5th-85th percentiles); overweight (BMI > 85th-95th percentiles); obesity (BMI > 95th percentile). Stunting was defined as height-for-age below the 3rd percentile of the NCHS/WHO reference [11].

A food frequency questionnaire (FFQ) that included 42 food items was developed, in which 25 of the items were taken from a questionnaire developed by Stene et al. for the West Bank and represented $40 \%$ of the energy intake at the household level [12]. Portion sizes were not included in the questionnaire. The students were asked, "How many times did you eat the following food items during the last week?" Scores in 5 categories were constructed by adding the weekly frequencies of the following items: animal foods (beef, turkey/chicken, liver, fish, hamburger, shawerma/kebab, canned meat, eggs); fruit and vegetables (fruits, fruit juice, salad, fresh and cooked vegetables); milk foods [milk, yogurt, white cheese, yellow cheese, lebneh (yogurt cheese)]; cookies (biscuits, cakes, chocolate); traditional foods [lentils, hummus (chickpea paste), falafel (deep-fried chickpea balls), fava beans].

The study was approved by the Ministry of Health and the Helsinki Ethical Committee in Gaza. In addition, ethical clearance was obtained from the Regional Medical Research Ethical Committee in Norway. Written consent was obtained from the adolescents' parents and the headmasters of the 10 schools.

\section{Statistical analysis}

SPSS for Windows, version 14.0 was used to analyse the data. Epi-Info 2002 was used to calculate the nutritional indices, including BMI-for-age percentiles and height-for-agepercentiles. Statistical significance was represented by a $P$ value $<0.05$. The chi-squared test was used to compare frequencies. Cross-tabulations and chi-squared tests were performed between all categorical dependent and independent variables. Bivariate and multivariate logistic regression models were used to estimate odds ratios (ORs) with their $95 \%$ confidence interval (CI) and to test for the significance of associations between risk factors and the school performance variable. Three models were constructed to assess the association between intake of fruit and vegetables and good school performance. In the first model we adjusted for the sociodemographic factors, in the second model we additionally adjusted for BMI-for-age percentile groups and in the third model we further adjusted for stunting.

\section{Results}

\section{Background characteristics}

Of the 1221 invited students, 932 were recruited ( $76.3 \%$ response rate). Of the 289 excluded, 25 withdrew, 63 were absent on the day of the interview, 70 parents refused on behalf of their children, 117 did not complete the questionnaire and 14 were outside the targeted age brackets. The final sample had a higher proportion of girls (53.9\%) than boys and a higher proportion of pupils with refugee status $(60.5 \%)$ than those with local citizen status. About $40 \%$ of the pupils lived in the high-income parts of Gaza city and $22.5 \%$ in the low-income area, while $15.3 \%$ of the sample lived in Jabalia village and 22.5\% in Jabalia refugee camp.

The prevalence of underweight was $5.4 \%$ and of overweight and obesity combined $18.5 \%$. The prevalence of stunting was $9.5 \%$. Overall $63.3 \%$ of the adolescents had good school performance (Table 1).

\section{Food intake and school performance}

More than two-thirds of the adolescents had a frequency intake of $<3$ times per week for several of the 5 categories of foods, including animal foods, fruit and vegetables, milk foods and traditional foods (Table 2). The unadjusted results of logistic regression revealed that school performance was positively associated with consumption of fruit and vegetables, cookies, soft drinks and rice. A borderline positive association was found between consumption of animal foods and school performance.

Intake of fruit and vegetables > 3 times/week had the strongest association with school performance compared with those consuming fruit and vegetables $\leq 3$ times/week. A higher proportion of adolescents with high fruit and vegetables consumption had good school performance $(72.6 \%$ versus $59.9 \%, P<0.001)$. Higher frequencies of consumption of soft drinks and rice were also significantly associated with good school performance, with OR of 1.69 (95\% CI: 1.14-2.52) and 1.57 (95\% CI: 1.17-2.11) respectively.

\section{Multivariate analysis}

In a multivariate model that included animal foods, fruit and vegetables, soft drinks, rice and cookies (Table 2), school performance remained strongly associated with the fruit and vegetables variable, while the relationship was only borderline for soft drinks and rice. After adjustment, scores for animal foods and for cookies were not independently associated with school performance.

In a multivariate analysis that adjusted for sociodemographic variables and nutritional status (Table 3), intake of fruit and vegetables was positively associated with good school performance. In the fully adjusted model, the OR for good school performance comparing a high with a low fruit and vegetables score was 1.61 (95\% CI: 1.11-2.32). Intake of rice and of soft drinks was not associated with good school performance in the fully adjusted model, and thus these 2 food items were taken out of the table (Table 3 ).

Stunting was negatively associated with good school performance. In comparison with those who were not stunted, stunted adolescents had 
an OR for good school performance of 0.53 (95\% CI: 0.31-0.90). BMI was not associated with school performance.

\section{Discussion}

This is the first study to examine associations between food intake and school performance among adolescents in Palestine. The results demonstrated an independent association between food intake, particularly of fruit and vegetables, and school performance among students in grades 7-9 in the northern part of the Gaza Strip. The association with fruit and vegetables intake appears robust, as it remained highly significant after adjustment for sociodemographic characteristics and nutritional status.

The analysed data were collected in 2002 as part of a larger research project entitled Lifestyles and Nutritional Status of School Adolescents in Gaza Strip. The sampling design was geared towards assessing sociodemographic differences in dietary pattern and nutritional status and not specifically on the relationship between diet and school performance. Even so, the study sample had sufficient variation in frequency intakes of different foods to analyse the association between those variables and school performance.

Since it was difficult to get information about household income in the research area we chose to create an index of household SES based on household possessions. The household SES was developed based on selected amenities which were listed among those used by the Palestinian Bureau of Statistics for SES assessment [13]. The WHO health behaviour in schoolaged children survey adopted a family affluence scale as an additional indicator to SES [14]. The same approach has been used in other studies and included similar items $[9,10,12,15,16]$. The developed SES score provided

\begin{tabular}{|c|c|c|}
\hline Variable & No. & $\%$ \\
\hline \multicolumn{3}{|l|}{ Sex } \\
\hline Girls & 502 & 53.9 \\
\hline Boys & 430 & 46.1 \\
\hline \multicolumn{3}{|l|}{ Residential status } \\
\hline Refugee & 561 & 60.5 \\
\hline Local citizen & 367 & 39.5 \\
\hline \multicolumn{3}{|l|}{ Residential area } \\
\hline Gaza city (high income) & 373 & 40.0 \\
\hline Gaza city (low income) & 210 & 22.5 \\
\hline Village & 143 & 15.3 \\
\hline Refugee camp & 206 & 22.1 \\
\hline \multicolumn{3}{|l|}{ Age (years) } \\
\hline 12 & 161 & 17.3 \\
\hline 13 & 291 & 31.2 \\
\hline 14 & 305 & 32.7 \\
\hline 15 & 175 & 18.8 \\
\hline \multicolumn{3}{|l|}{ Father's work status } \\
\hline Unemployed & 429 & 46.1 \\
\hline Employed & 501 & 53.9 \\
\hline \multicolumn{3}{|l|}{ Household SES } \\
\hline Low & 244 & 26.9 \\
\hline Medium & 431 & 47.5 \\
\hline High & 232 & 25.6 \\
\hline \multicolumn{3}{|l|}{ Father's education } \\
\hline Low & 485 & 52.7 \\
\hline Medium & 262 & 28.5 \\
\hline High & 173 & 18.8 \\
\hline \multicolumn{3}{|l|}{ Mother's education } \\
\hline Low & 531 & 57.6 \\
\hline Medium/high & 391 & 42.4 \\
\hline \multicolumn{3}{|l|}{$B M I^{a}$} \\
\hline Underweight & 50 & 5.4 \\
\hline Normal & 710 & 76.2 \\
\hline Overweight/obese & 172 & 18.5 \\
\hline \multicolumn{3}{|l|}{ Stunting ${ }^{b}$} \\
\hline Non-stunted & 843 & 90.5 \\
\hline Stunted & 89 & 9.5 \\
\hline Good school performance & 590 & 63.3 \\
\hline
\end{tabular}

aUnderweight: $B M I$-for-age < 5th percentile; overweight and obesity: > 85th percentile. ${ }^{b}$ Stunting: height-for-age $<3$ rd percentile.

$n=$ number of respondents; $t / w=$ times per week; SES = socioeconomic status; $B M I=$ body mass index .

convincing results; the correlation coefficient between the household SES score and father's employment status was 0.40 and father's educational level was $0.32(P=0.01)$.
The present study had certain limitations. The FFQ was not validated, but the food list was constructed with a thorough knowledge of the eating patterns in Gaza and with the help of 


\begin{tabular}{|c|c|c|c|c|c|c|c|}
\hline \multirow[t]{2}{*}{ Variable } & \multirow[t]{2}{*}{ No. } & \multicolumn{2}{|c|}{$\begin{array}{l}\text { Good school } \\
\text { performance }\end{array}$} & \multicolumn{2}{|c|}{$\begin{array}{l}\text { Bivariate logistic } \\
\text { regression }\end{array}$} & \multicolumn{2}{|c|}{$\begin{array}{l}\text { Multivariate logistic } \\
\text { regression }\end{array}$} \\
\hline & & $\%$ & $P$-value & OR $(95 \% \mathrm{Cl})$ & $P$-value & OR $(95 \% \mathrm{Cl})$ & $P$-value \\
\hline \multicolumn{8}{|c|}{ Animal foods } \\
\hline$\leq 3 \mathrm{t} / \mathrm{w}$ & 622 & 61.3 & 0.07 & 1 & & 1 & \\
\hline$>3 \mathrm{t} / \mathrm{w}$ & 310 & 67.4 & & $1.31(0.89-1.74)$ & 0.07 & $1.0(0.72-1.37)$ & 0.48 \\
\hline \multicolumn{8}{|c|}{ Fruit \& vegetables } \\
\hline$\leq 3 \mathrm{t} / \mathrm{w}$ & 680 & 59.9 & $<0.001$ & 1 & & 1 & \\
\hline$>3 \mathrm{t} / \mathrm{w}$ & 252 & 72.6 & & $1.78(1.29-2.44)$ & $<0.001$ & $1.50(1.05-2.14)$ & 0.02 \\
\hline \multicolumn{8}{|l|}{ Cookies } \\
\hline$\leq 3 \mathrm{t} / \mathrm{w}$ & 470 & 59.4 & 0.01 & 1 & & 1 & \\
\hline$>3 \mathrm{t} / \mathrm{w}$ & 462 & 67.3 & & $1.41(1.08-1.84)$ & 0.01 & $1.12(0.83-1.51)$ & 0.44 \\
\hline \multicolumn{8}{|l|}{ Milkfoods } \\
\hline$\leq 3 \mathrm{t} / \mathrm{w}$ & 718 & 62.8 & 0.63 & 1 & & - & - \\
\hline$>3 \mathrm{t} / \mathrm{w}$ & 214 & 65.0 & & $1.10(0.80-1.51)$ & 0.59 & - & - \\
\hline \multicolumn{8}{|c|}{ Traditional foods } \\
\hline$\leq 3 \mathrm{t} / \mathrm{w}$ & 655 & 62.7 & 0.60 & 1 & & - & - \\
\hline$>3 \mathrm{t} / \mathrm{w}$ & 277 & 64.6 & & $1.08(0.81-1.45)$ & 0.59 & - & - \\
\hline \multicolumn{8}{|l|}{ Soft drinks } \\
\hline$\leq 3 \mathrm{t} / \mathrm{w}$ & 791 & 61.6 & 0.01 & 1 & & 1 & \\
\hline$>3 \mathrm{t} / \mathrm{w}$ & 141 & 73.0 & & $1.69(1.14-2.52)$ & 0.01 & $1.33(0.87-2.04)$ & 0.05 \\
\hline \multicolumn{8}{|l|}{ Rice } \\
\hline$\leq 3 \mathrm{t} / \mathrm{w}$ & 633 & 60.0 & 0.003 & 1 & & 1 & \\
\hline$>3 \mathrm{t} / \mathrm{w}$ & 299 & 70.2 & & 1.57 (1.17-2.11) & 0.003 & $1.36(1.00-1.85)$ & 0.05 \\
\hline \multicolumn{8}{|c|}{ Tea \& coffee } \\
\hline$\leq 21 \mathrm{t} / \mathrm{w}$ & 781 & 64.0 & 0.31 & 1 & & - & - \\
\hline$>21 \mathrm{t} / \mathrm{w}$ & 151 & 59.6 & & $0.83(0.58-1.18)$ & 0.30 & - & - \\
\hline
\end{tabular}

$n=$ number of respondents; $t / w=$ times per week; OR=odds ratio; $C l=$ confidence interval.

previous evidence about dietary patterns and energy intake in the West Bank [12]. One limitation was that the intake of tomatoes and cucumber was not included in the questionnaire. These vegetables are traditionally eaten partially as snacks and partially at some meals in small amounts. It is thus difficult to obtain a precise estimation of this intake in a FFQ without portion sizes. However, a recent study from the West Bank showed that daily consumption of tomatoes and cucumbers was positively associated with SES [16]. It is also possible that those with low SES over-reported fruit and vegetables intake due to social desirability. However, this over-reporting would underestimate the association between fruit and vegetables and school performance given the positive association between SES and school performance. Another limitation is related to recall bias, which is common and is difficult to overcome in FFQ and food records studies [17], especially when portion sizes are included and absolute nutrient intakes of each serving are to be estimated. However, our main focus was only on the number of daily or weekly intake of foods listed in the FFQ. Therefore recall bias would be less of a concern.

Our finding of an association between fruit and vegetables intake and school performance is consistent with a recent finding from Canada [2]. In that study, higher intake of fruit and vegetables was positively associated with a higher level of school performance in both boys and girls. Several mechanisms could link fruit and vegetables intake and school performance, but these are yet to be elucidated. For example, a deficiency in micronutrients could play a part; it is known that intake of fruit and vegetables prevent anaemia and micronutrient deficiency [18]. Anaemia affects more than one-third of the population in Gaza $[9,19]$, and anaemic children are known to have poorer learning outcomes [20]. Iodine is also an important micronutrient, and deficiencies of this element can be quite serious. A recent study showed that about $15 \%$ of school-aged children in Gaza aged 8-10 years had grade 1 or grade 2 goitre [19]. This deficiency occurs despite the fact that Gaza borders 


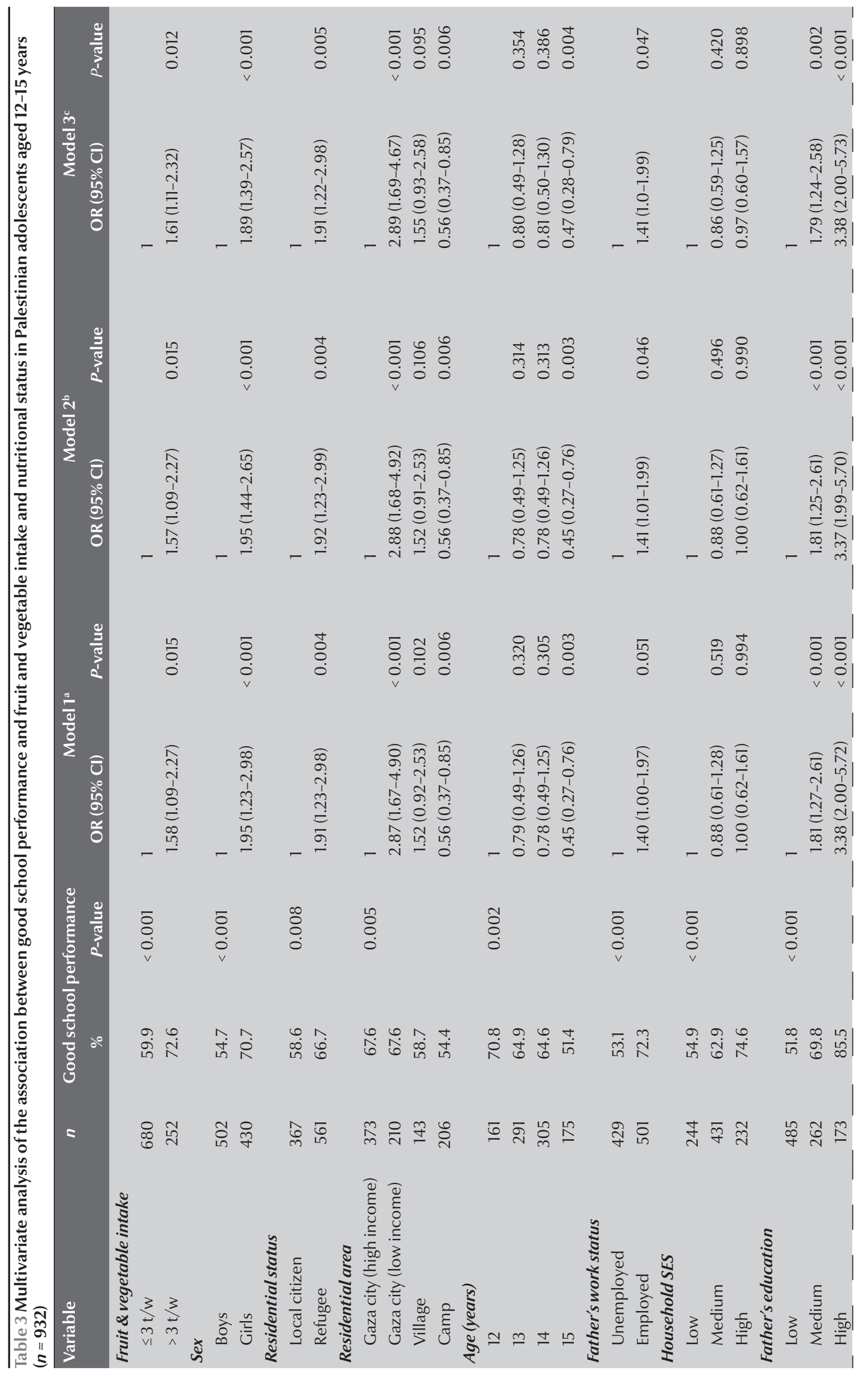




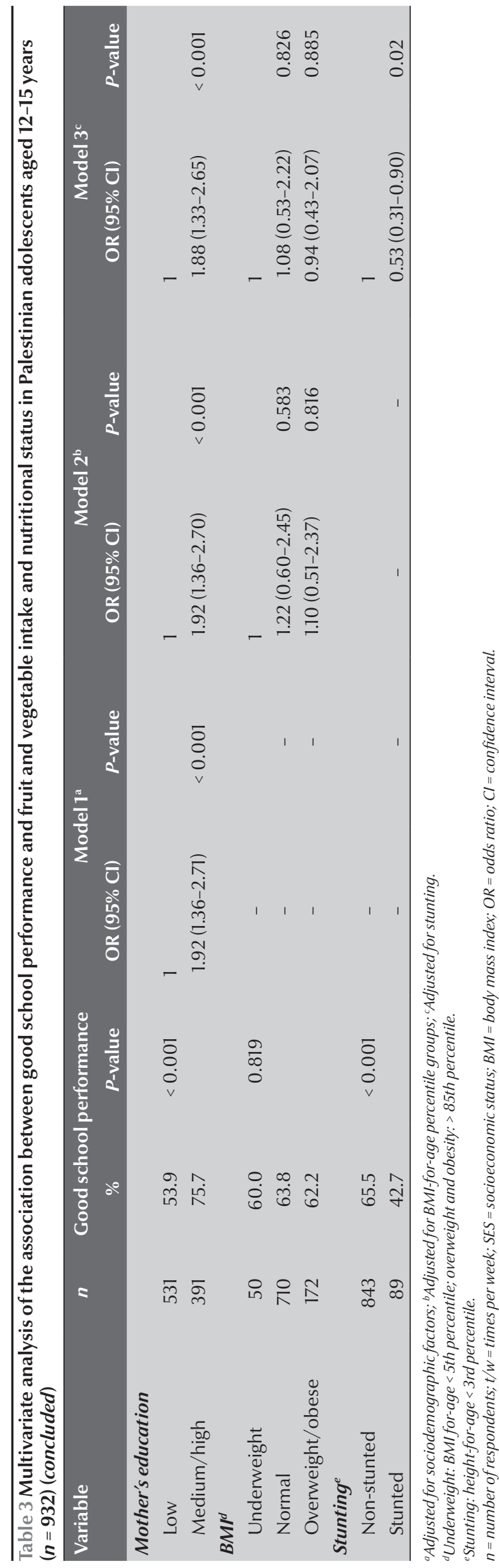

the sea. However, at the time of the study fishing was banned by the Israeli government for security reasons. Furthermore, $22 \%$ of the reported cases of rickets in this Gaza population involved deficiencies of both vitamins A and D [19].

Abudayya et al. found that stunting was a public health problem among schoolboys in the Gaza Strip [9]. In the present study we found an inverse association between stunting and school performance, which is consistent with current knowledge that stunting lowers cognitive capacity $[4,21]$. Adjustment for BMI and stunting did not change the association between fruit and vegetables and school performance. This may suggest that the association is independent of general nutritional status.

The association between fruit and vegetables and school performance could be confounded by SES, and in the study population SES was positively associated with fruit and vegetables intake. Furthermore, it is known that SES is positively related to school performance $[3,22]$. However, the association between fruit and vegetables and school performance was still significant after adjusting for SES in the multivariate analysis.

Fruit and vegetables intake is influenced by a variety of lifestyle factors, including smoking, drinking and physical activity and all of these factors are related to school performance $[2,23]$. In our study, however, we were not able to control for these factors and further research is therefore needed.

Fruit and vegetables such as oranges, strawberries, tomatoes and cucumbers are usually available in the local market and sometimes at very low prices, but even so, many residents of the Gaza Strip are unable to afford them [24]. In the present study, we found that less than two-thirds of the adolescents ate fruit and vegetables more than 3 times per week. The intake of cucumbers and tomatoes was not included in the questionnaire; however a recent study from the West Bank found that about $40 \%$ of respondents did not eat these items daily. We might thus conclude that adolescents of low SES did not meet the recommendation consumption of 5 portions of fruit and vegetables per day [25].

Our study revealed a strong negative association between stunting and good school performance but no association between BMI and school performance; the latter being consistent with results from other studies $[2,26]$. On the other hand, some studies have noted a consistent association between childhood overweight and poorer school performance [27]. We also found an association between school performance and both the family's SES and the parents' education. This finding supports our confidence in our measurement of school performance. Our finding that boys performed less well academically than did girls is in line 
with previous research demonstrating gender variations in performance $[2,28]$.

In conclusion, intake of fruit and vegetables was positively associated with school performance among adolescents in Gaza Strip while stunting was negatively associated with school performance. This provides additional evidence that school performance is strongly affected by the dietary quality and the nutritional status of the adolescents. The findings of this study support a broader implementation of school nutrition programmes that may improve students' diets, their academic performance, and, over the long term, their health.

\section{Acknowledgements}

This study is a collaborating project between Palestinian Ministry of Health and the University of Oslo. The authors would thank Norwegian Directorate for Health, Section for Nutrition for their financial support and the Palestinian Ministry of Health for facilitating the field work.

\section{References}

1. Grantham-McGregor S. Can the provision of breakfast benefit school performance? Food and Nutrition Bulletin, 2005, 26(2 Suppl. 2):S144-158.

2. Florence MD, Asbridge M, Veugelers PJ. Diet quality and academic performance. Journal of School Health, 2008, 78:209215.

3. Kramer RA, Allen L, Gergen PJ. Health and social characteristics and children's cognitive functioning: results from a national cohort. American Journal of Public Health, 1995, 85:312-318.

4. Ivanovic D et al. Nutrition and education. III. Educational achievement and food habits of Chilean elementary and high school graduates. Archivos Latinoamericanos de Nutricion, 1992, 42:9-14.

5. Galler JR, Ramsey F. A follow-up study of the influence of early malnutrition on development: behavior at home and at school. Journal of the American Academy of Child and Adolescent Psychiatry, 1989, 28:254-261.

6. Grantham-McGregor S. A review of studies of the effect of severe malnutrition on mental development. Journal of Nutrition, 1995, 125(Suppl.);2233S-2238S.

7. Guidelines for school health programs to promote lifelong health eating. Atlanta, Georgia, Centers for Disease Control and Prevention, 1996 (Report No. RR-9).

8. Kaufmann NA, Poznanski R, Guggenheim K. Teen-agers dieting for weight control. Annals of Nutrition and Metabolism, 1974, 16:30-37.

9. Abudayya A et al. Overweight, stunting, and anemia are public health problems among low socioeconomic groups in school adolescents (12-15 years) in the North Gaza Strip. Nutrition Research, 2007, 27:762-771.

10. Abudayya AH et al. Sociodemographic correlates of food habits among school adolescents (12-15 year) in North Gaza Strip. BMC Public Health, 2009, 9:185.

11. Kuczmarski RJ et al. 2000 CDC Growth Charts for the United States: methods and development. Vital Health Statistics, 2002, 11(246):1-190.

12. Stene LC et al. Food consumption patterns in a Palestinian West Bank population. European Journal of Clinical Nutrition, 1999, 53:953-958.

13. Demographic health survey, 2004: final report. Ramallah, Palestinian Central Bureau of Statistics, 2006.

14. Currie CE et al. Indicators of socioeconomic status for adolescents: the WHO Health Behaviour in School-aged Children Survey. Health Education Research, 1997, 12:385-397.
15. Shi $Z$ et al. The sociodemographic correlates of nutritional status of school adolescents in Jiangsu Province, China. Journal of Adolescent Health, 2005, 37:313-322.

16. Miki $\mathrm{N}$ et al. Dietary habits of Palestinian adolescents and associated socio-demographic characteristics in Ramallah, Nablus and Hebron governorates. Public Health Nutrition, 2010, 13(9):1419-1429.

17. Livingstone MB, Robson PJ, Wallace JM. Issues in dietary intake assessment of children and adolescents. British Journal of Nutrition, 2004, 92 (Suppl. 2):S213-S222.

18. Nobmann ED et al. The diet of Alaska Native adults: 1987-1988. American Journal of Clinical Nutrition, 1992, 55:1024-1032.

19. Comprehensive food security and vulnerability analysis (CFSVA): West Bank and Gaza Strip. Jerusalem, Food and Agriculture Organization of the United Nations (FAO), 2007.

20. Otero GA et al. Psychological and electroencephalographic study in school children with iron deficiency. International Journal of Neuroscience, 1999, 99:113-121.

21. Fu ML et al. Association between unhealthful eating patterns and unfavorable overall school performance in children. Journal of the American Dietetic Association, 2007, 107:1935-1943.

22. Patterson CJ, Kupersmidt JB, Vaden NA. Income level, gender, ethnicity, and household composition as predictors of children's school-based competence. Child Development, 1990, 61:485-494.

23. Daley AJ, Ryan J. Academic performance and participation in physical activity by secondary school adolescents. Perceptual and Motor Skills, 2000, 91:531-534.

24. Abdeen Z et al. Nutritional assessment of the West Bank and Gaza Strip. Jerusalem, Ministry of Health, Al Quds University, US Agency for International Development and Care International, 2003.

25. Stelle BF, Clayton MM, Tucker RE. Role of breakfast and of between-meal foods in adolescents' nutrient intake. Journal of the American Dietetic Association, 1952, 28:1054-1057.

26. Li Y et al. Overweight is associated with decreased cognitive functioning among school-age children and adolescents. Obesity, 2008, 16:1809-1815.

27. Taras H, Potts-Datema W. Obesity and student performance at school. Journal of School Health, 2005, 75:291-295.

28. Considine G, Zappalà G. The influence of social and economic performance disadvantage in the academic performance in the school students in Australia. Journal of Sociology, 2002, 38:129-148. 